Ann. Biol. anim. Bioch. Biophys., 1978, 18 (4), 1083-1088.

\title{
Conservation à court terme des gamètes de Salmonidés à des températures voisines de $0{ }^{\circ} \mathrm{C}$
}

par P. CARPENTIER, R. BILLARD

Laboratoire de Physiologie des Poissons, I. N. R. A.

78350 jouy en Josas, France

Summary. Shorf term preservation of gametes in Salmonids.

Preservation of gametes was attempted in two species of salmonids brown trout (Salmo trutta) and rainbow trout (Salmo gairdneri). Tests were carried out with gametes stored at $0^{\circ} \mathrm{C}$ during 7 days. The following results were obtained : after one day a drop of fertilizability (13 p. 100) was observed for ova in brown trout but not in rainbow trout. However, after 5 days the fertilizability of the female gametes is the same for both species (about 57 p. 100). Ova of brown trout vary more widely in response to aging than those of rainbow trout. The loss of ova fertilizability may be partly compensated by using larger doses of sperm $\left(12.10^{6}\right.$ spermatozoa/egg). Sperm of rainbow trout lose 25 p. 100 of their fertilizing ability after one day and 10 p. 100/day during the following days. There is a significant interaction between aging of sperm and ova which is not of a simple type additif or multiplicatif.

\section{Introduction.}

La conservation des gamètes a de nombreux avantages notamment leur transport lorsque les lieux de prélèvements et d'insémination sont différents, la facilité du travail en pisciculture ou des améliorations génétiques (croisements, hybridation). Pour le sperme, les possibilités de conservation sont de deux ordres :

- congélation à $-190^{\circ} \mathrm{C}$ qui permet une conservation à long terme et qui est possible pour des poissons marins (Billard et Dupont, 1975), mais très aléatoire pour les salmonidés (Stoss et al., 1978, Billard, 1978) ;

- conservation à court terme à des températures voisines de $00^{\circ} \mathrm{C}$ dans des milieux divers : colloïdes (Bratanov et Dikov, 1961), DMSO et éthylène glycol (Truscott ef al., 1968 ; Sanchez-Rodriguez et Billard, 1977).

Pour les ovules, l'aptitude à être fécondés peut se prolonger chez les salmonidés pendant au moins 8 jours (Escaffre ef al., 1977 ; Bry, communication personnelle), mais après prélèvement, la durée de la fécondabilité est variable et dépend de facteurs variés comme la température, la lumière, le milieu de conservation (Takano et al., 1973). 
Dans le présent travail, les possibilités de conservation des ovules et du sperme à des températures voisines de $0^{\circ} \mathrm{C}$ seront testées en prenant pour critère de survie l'aptitude à la fécondation pour les ovules et le pouvoir fécondant et la motilité pour le sperme. En outre, l'évolution de la composition minérale $\left(\mathrm{Na}^{+}, \mathrm{K}^{+}\right) \mathrm{du}$ liquide séminal, déjà étudiée chez le Saumon (Hwang et Idler, 1969) sera examinée pendant la période de conservation.

\section{Matériel et méthodes.}

Matériel animal. - L'étude porte sur la Truite Arc-en-ciel (Salmo gairdneri) et la Truite fario (Salmo trutta). Les géniteurs d'un poids de 300 à $400 \mathrm{~g}$ proviennent de piscicultures privées. Les femelles fario avaient ovulé spontanément à une date indéterminée avant leur arrivée au laboratoire qui s'est effectuée une semaine avant le début de l'expérience ; l'ovulation des femelles Arc-en-ciel a été induite par traitement hormonal ( $\mathrm{t}-\mathrm{GTH}, 0,1 \mathrm{r} / \mathrm{g}$ poids vif) et les ovules destinés à l'expérimentation ont été prélevés $3 \mathrm{j}$ après l'ovulation. Les mâles utilisés sont pris dans un groupe d'individus isolés au début de l'expérience et en spermiation depuis environ un mois.

Effets du vieillissement des gamètes sur le pourcentage de fécondation. - Au début de l'expérience, les ovules sont prélevés sur plusieurs femelles (10 truites Arc-en-ciel et 3 fario) et placés séparément en boîtes plastiques de $400 \mathrm{ml}$ avec le liquide coelomique homologue ( $10 \mathrm{ml}$ de fraction de 200 ovules). Pour chaque mâle, $3 \mathrm{ml}$ de sperme sont placés en tubes à hémolyse de $5 \mathrm{ml}$ maintenus bouchés. Les gamètes ainsi conditionnés sont amenés de la température ambiante $\left(10-12{ }^{\circ} \mathrm{C}\right)$ à la température de con servation $\left(0^{\circ} \mathrm{C}\right)$ en $20 \mathrm{mn}$, puis stockés à l'obscurité en étude réfrigérée. La fertilité des ovules et le pouvoir fécondant du sperme sont appréciés à des temps croissants : $0,1,3,5$ et 7 jours après prélèvement ; les ovules sont inséminés par lots de 200 environ additionnés de $10 \mathrm{ml}$ de dilueur avec un mélange de sperme frais provenant de plusieurs mâles ; d'autre part, le pouvoir fécondant du sperme vieilli est testé sur des lots également de 200 ovules provenant du mélange de la production de 3 à 5 femelles fraîchement ovulées. En vue de tester les effets cumulés du vieillissement des gamètes mâles et femelles sur le pourcentage de fécondation, on a mis en présence à $J_{1}, J_{3}, J_{5}$ et $J_{7}$ des ovules et du sperme conservés dans les conditions définies ci-dessus. Dans chaque cas, l'insémination est pratiquée à $+4^{\circ} \mathrm{C}$ à deux taux de dilution différents $\left(4.10^{-3}\right.$ et $\left.2.10^{-4}\right)$, selon la méthode décrite par Billard et al. (1974) en mettant en œuvre le dilueur 532 (Billard, 1977). Après insémination, les œufs sont mis en incubation en eau recyclée et thermorégulée à $10 \pm 0,5^{\circ} \mathrm{C}$ et le pourcentage d'œufs embryonnés (stade œillé) qui représente une estimation par défaut du taux de fécondation est établi à 200 degré-jours. En outre, le pourcentage d'individus survivants est dénombré en fin de résorption vitelline.

Effets du vieillissement du sperme sur la durée de motilité ef le rapport $\mathrm{Na}^{+} / \mathrm{K}^{+}$. - La conservation du sperme est d'autre part testée d'après l'évolution de la concentration en $\mathrm{Na}^{+}$et $\mathrm{K}^{+}$mesurée par spectrophotométrie de flamme dans le liquide séminal et d'après la durée de motilité. Cette dernière est définie comme étant la durée pendani laquelle la motilité observée sous microscope se maintient à son intensité initiale (après dilution du sperme dans un iso-volume de dilueur 532). Dans cette expérience, le 
sperme de 10 mâles truite Arc-en-ciel est prélevé séparément et conservé pendant 6 jours dans les conditions définies ci-dessus. Les mesures de motilité, pouvoir fécondant et $\mathrm{Na}^{+}-\mathrm{K}^{+}$sont pratiquées à $\mathrm{J}_{0}$ ef $\mathrm{J}_{6}$.

Analyse statistique. - Les résultats sont testés par analyse de variance avec répétition en modèle factoriel à effets fixes. Dans les cas de pourcentages, la transformation angulaire a été utilisée pour stabiliser la variance résiduelle (Snedecor, 1956).

\section{Résultats.}

\section{Conservation des ovules.}

Chez la Truite Fario (fig. 1) l'aptitude des ovules à être fécondés (dilution de 4.10-3) diminue significativement $(P<0,01)$ de 13 p. 100 après $24 h$ (passage de 73 à 60 p. 100), puis se maintient à ce même niveau jusqu'à la fin de l'expérience ( 7 jours) ; les mêmes résultats sont observés à la dilution de $2 \cdot 1^{-4}$. La mortalité embryonnaire mesurée en fin de résorption vitelline et exprimée en pourcentage par rapport au nombre d'œufs œillés est significativement plus élevée $(P<0.025)$ dans les lots d'œufs conservés 7 jours $\left(J_{7}\right)$ que dans les lots de départ $\left(J_{0}\right)$.

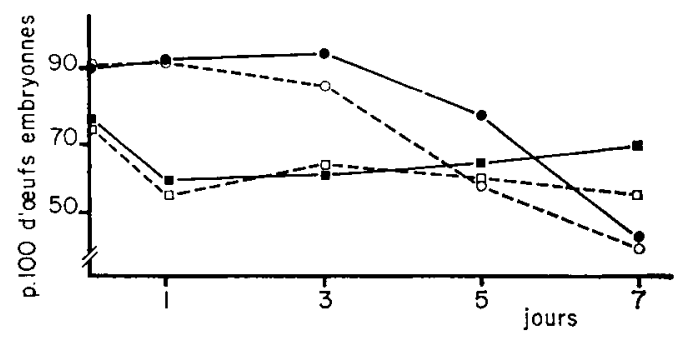

FIG. 1.

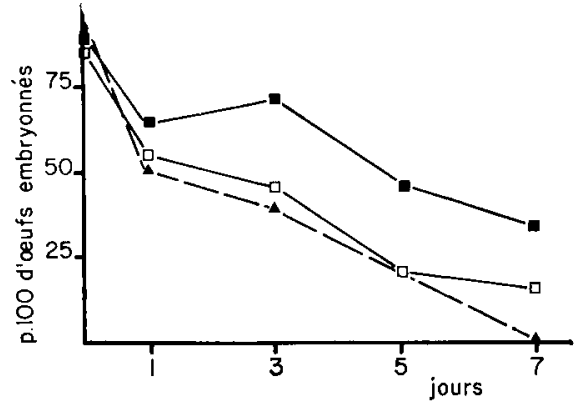

FIG. 2.

FIG. 1. - Effet de la conservation à $0^{\circ} \mathrm{C}$ dans le liquide coelomique et à l'obscurité d'ovules de truite forio (o) et arc-en-ciel (), sur leur aptitude à être fécondé, estimée d'après le pourcentage d'œưfs embryonnés à 200 degré-jours. Taux de dilution du sperme lors de l'insémination : 4.10-3 $2.10^{-4} \ldots . .$.

FIG. 2. - Evolution du pouvoir fécondant des gamètes de truites arc-en-ciel conservés pendant 7 jours à $0^{\circ} \mathrm{C}$ ef à l'obscurité. - Sperme vieilli et ovules frais lors de l'insémination : a 4.10-3 (soit 1,2 million de spermatozoïdes/ovules; a $2.10^{-4}$ (soit 20.000 spermatozoïdes par ovules). — Insémination d'ovules vieillis avec du sperme vieilli $\triangle$ taux de dilution $4.10^{-3}$.

Pour la Truite Arc-en-ciel, il n'y a pas de diminution de l'aptitude à la fécondation pendant les trois premiers jours de conservation (fig. 1). Entre $J_{3}$ et $J_{7}$, il y a par contre une diminution significative $(P<0,01)$ du pourcentage d'œufs embryonnés. $A J_{3}$ et $J_{5}$, on observe un effet favorable des faibles dilutions en spermatozoïdes qui compense les effets du vieillissement. Entre $J_{0}$ et $J_{7}$ il n'y a pas de différences dans les taux de survie post-embryonnaire (entre éclosion et résorption). 
Conservation du sperme de Truife Arc-en-ciel.

- Evolution du pouvoir fécondant (fig. 2). - Le pouvoir fécondant chute brutalement de 25 p. 100 après $24 \mathrm{~h}$ de conservation. Au cours des jours suivants, la chute journalière est moins importante (de l'ordre de 10 p. 100). En outre, le pouvoir fécondant varie selon la dilution : au taux le plus élevé $\left(2,10^{-4}\right)$ la chute du pouvoir fécondant est significativement $(P<0,01)$ plus forte de 10 à 25 p. 100 par rapport aux faibles dilutions $\left(4,10^{-3}\right)$.

- Vieillissement cumulé des gamètes. - Lorsque les ovules et les spermatozoïdes ont vieilli pendant 7 jours, le taux de fécondation (fig. 2), toujours mesuré à 200 degréjours, diminue plus fortement que lorsque le vieillissement porte seulement sur les ovules ou le sperme séparément (fig. 1 et fig. 2 pour le sperme). Il existe une interaction significative entre le vieillissement du sperme et celui des ovules, mais d'après le test $\chi^{2}$ il ne s'agit pas d'un mode simple de type additif ou multiplicatif.

- Relation entre pouvoir fécondant, durée de motilité et rapport $\mathrm{Na}^{+} / \mathrm{K}^{+}$(tabl. 1). Après 6 jours de conservation, le pouvoir fécondant du sperme et la durée de motilité sont significativement diminués $(P<0,005)$. Le rapport $\mathrm{Na}^{+} / \mathrm{K}+$ du liquide séminal ne présente pas de variations notables pendant cette période. II n'y a pas cependant de relations significatives entre durée de motilité et pouvoir fécondant à l'intérieur de chacun des groupes $\left(J_{0}\right.$ ou $\left.J_{6}\right)$. Il apparaît d'autre part que pour des spermes dont les durées de motilité sont de 35 à 50 sec., le pouvoir fécondant est d'environ 70 p. 100 à $J_{0}$ et 15 p. 100 seulement à $J_{6}$, de sorte que la durée de motilité ne rend pas compte de tous les phénomènes de vieillissement qui affectent le sperme.

\section{TABLEAU 1}

Appréciation par différents critères de la qualité du sperme lors du prélèvement $\left(J_{0}\right)$ et après conservation pendant $6 j\left(\mathrm{~J}_{6}\right)$ d $0^{\circ} \mathrm{C}$

Entre parenthèses : nombre d'individus. Comparaison statistique entre $J_{0}$ et $J_{6}$ par analyse de variance

\begin{tabular}{|c|c|c|c|c|c|}
\hline $\begin{array}{l}\text { Critères d'appréciation } \\
\text { de la qualité du sperme }\end{array}$ & \rfloor$_{0}$ & & $\mathbf{J}_{6}$ & & $\begin{array}{c}\text { Comparaison } \\
\text { statistique }\end{array}$ \\
\hline $\begin{array}{l}\text { Durée de la motilité (en sec.) } \ldots \ldots \\
\text { Pourcentage de fécondation } \ldots \ldots \\
\text { Rapport } \mathrm{Na} / \mathrm{K}^{+} \ldots \ldots \ldots \ldots \ldots \ldots\end{array}$ & $\begin{array}{l}53 \pm 6,04 \\
72 \pm 2,15 \\
1,22\end{array}$ & $\begin{array}{l}(10) \\
(10) \\
(10)\end{array}$ & $\begin{array}{c}26 \pm 6,92 \\
11 \pm 2,40 \\
0,87\end{array}$ & $\begin{array}{l}(9) \\
(9) \\
(8)\end{array}$ & $\begin{array}{l}P<0,005 \\
P<0,005\end{array}$ \\
\hline
\end{tabular}

\section{Discussion.}

Des ovules de truite extraits de la cavité générale ont été conservés à des températures voisines de $0^{\circ} \mathrm{C}$ pendant 3 jours au moins pour les truites Arc-en-ciel chez lesquelles les œufs étaient fraîchement ovulés. La chute de l'aptitude à la fécondation des ovules de truite Fario est plus rapide (diminution significative après $24 \mathrm{~h}$ de conservation), mais dans ce cas, les ovules avaient séjournés dans la cavité générale depuis plus de 8 jours. Il faut noter néanmoins qu'après 7 jours de conservation in vitro la fécondabilité des ovules de truite Fario est plus élevée que celle des ovules de truite 
Arc-en-ciel, bien que le temps écoulé depuis l'ovulation soit plus court. Le vieillissement se traduit en outre, chez la truite Fario, par une mortalité plus élevée pendant la période de résorption, aucun effet significatif n'ayant été décelé pour la truite Arcen-ciel. Cette différence peut à nouveau être mise en relation avec la durée totale du vieillissement (in vivo + in vitro) après l'ovulation qui est plus longue dans le cas de la truite Fario. Cela montre d'autre part que le pourcentage de fécondation ou le pourcentage d'œufs embryonnés ne rend pas totalement compte de la qualité des ovules puisque des mortalités se produisent pendant la résorption vitelline pour les lots vieillis.

D'une façon plus générale, les durées de conservation des ovules de Salmonidés rapportées dans la littérature sont très variables et de nombreux facteurs peuvent expliquer les différences entre auteurs. Des durées de conservation des ovules de $24 \mathrm{~h}$ pour S. gairdneri (Hamor, 1966) et de 2 à 4 jours pour O. nerka (Barrett, 1951 ; Foerster, 1965) ont été rapportées. La durée de conservation est de quelques jours dans le liquide coelomique (Ginsburg, 1968; Takano et al.,1973), et n'est que de quelques heures à sec (Billard, 1976). D'autres facteurs comme la température sont aussi importants (Withler et Morley, 1968). Il est possible de compenser au moins dans le cas de la truite Arc-en-ciel les effets du vieillissement sur la fécondabilité des ovules en augmentant le nombre de spermatozoïdes lors de l'insémination.

Dans les conditions de la présente expérience, la durée de conservation du sperme reste relativement brève (diminution significative du pouvoir fécondant après $24 \mathrm{~h}$ ). Pour obtenir des durées plus longues, il est nécessaire d'avoir recours à des procédés plus complexes : soil addition de cryoprotecteurs (Sanchez-Rodriguez et Billard, 1977), soit mise sous atmosphère d'oxygène (Stoss ef al., 1978). Dans ces deux cas, la température de stockage était inférieure à $0^{\circ} \mathrm{C}$ (respectivement -4 et $-1{ }^{\circ} \mathrm{C}$ ). Lors du vieillissement, le pouvoir fécondant du sperme chute beaucoup plus rapidement que la durée de motilité (łabl. 1) et, à l'intérieur de chaque groupe $J_{0}$ ef $J_{6}$, il n'y a pas de corrélation entre ces deux paramètres. La durée de motilité n'est donc pas en mesure de constituer un bon critère d'appréciation du pouvoir fécondant, excepté dans les cas où le sperme est non motile car alors, il n'est jamais fécondant. Cependant des motilités faibles et brèves peuvent conduire à quelques fécondations, mais à condition que le taux de dilution ne soit pas trop élevé ; on observe d'autre part que le pouvoir fécondant du sperme est amélioré lorsque le nombre de spermatozoïdes (fig. 2) utilisé lors de l'insémination est augmenté ; la quantité moyenne de spermatozoïdes par ovule est ainsi plus élevée ef la probabilité que le micropyle reçoive un spermatozoïde est plus grande, compensant de la sorte, au moins partiellement, la perte de motilité en durée et en intensité. Ce n'est donc que dans des cas limites et seulement dans un sens négatif que le critère durée de motilité peut être utilisé en pratique salmonicole : il n'est pas possible de sélectionner des spermes à haut pouvoir fécondant, mais il est possible d'éliminer ceux dont le pouvoir fécondant est faible.

Symposium sur la Reproduction des Poissons Paimpont, France, 19-21 septembre 1977.

Remerciements. - Ce travail a été partiellement financé par le Ministère de l'Environnement et de la Culture (contrat no 37-76). 


\section{Références}

BARRETT I., 1951. Fertility of salmonid eggs and sperm after storage. J. Fish. Res. Bd. Can., 8, 125-133 BILLARD R., 1978. Some data on gametes preservation and artificial insemination in teleost fish Coll. Aquac. Thon., Sète, CNEXO éd. (sous presse).

BILLARD R., DUPONT J., 1975. Etudes sur la congélation du sperme de poissons marins (bar, daurade, turbot). C. R. Activité CNEXO, 15 PP.

BILLARD R., PETIT J., JALABERT B., SZÖLLÖSI D., 1974. Artificial insemination in trout using a sperm diluant, 715-723. In BLAXTER J. H. S., The early life history of fish. Springer Verlag Berlin, Heidelberg, New York.

BILLARD R., 1976. Effet de l'agitation des ovules et des œufs de truite Arc-en-ciel sur la fécondabilité et le début du développement embryonnaire. Bull. fr. Piscic., 262, 5-11.

BILLARD R., 1977. Utilisation d'un système fris-glycocolle pour tamponner le dilueur d'insémination de truite. Bull. fr. Piscic., 264, 102-112.

BRATANOV C., DIKOV V., 1961. Certaines particularités du sperme chez les poissons. $4^{e}$ Congr. int. Reprod. anim., La Haye, 895-897.

FOERSTER R. E., 1965. Effect of retention of spermatozoa and ova of sockeye salmon, Oncorhynchus nerka, in water and without addition of water, on fertility. J. Fish. Res. Bd. Can., 22, 1503-1521.

ESCAFFRE A. M., PETIT J., BILLARD R., 1977. Evolution de lo quantité d'ovules récoltés et conservation de leur aptitude à être fécondés au cours de la période post-ovulatoire chez la truite Arc-en-ciel. Bull. fr. Piscic., 265, 134-142.

GINSBURG A. S., 1968. Fertilization in fishes and the problem of polyspermy. Akad. Nauk. USSR, NOAA and Nat. Sci. Found. translation.

HAMOR T., 1966. A study of the genital products of trout species S. trutta. and S. irrideus (en hongrois). Magyar Allat Kozl., 43, 63-68.

HWANG P. C., IDLER D. R., 1969. A study of major cation, osmotic pressure and $\mathrm{pH}$ in seminal components of Atlantic Salmon. J. Fish. Res. Bd. Can., 26, 413-419.

SANCHEZ-RODRIGUEZ M., BILLARD R., 1977. Conservation de la motilité et du pouvoir fécondant, du sperme de truite Arc-en-ciel maintenu à des températures voisines de $0^{\circ} \mathrm{C}$. Bull. fr. Piscic. 265, $143-152$.

SNEDECOR G. W., 1956. Statistical methods. lowa State Univ. Press., Ames, lowa.

STOSS J., BÜYÜKHATIPOGLU S., HOLTZ W., 1978. Short-term and cryopreservation of rainbow trout (Salmo gairdneri) sperm. Ann. Biol. anim. Bioch. Biophys., 18, 1077-1082.

TAKANO K., HIROI O., YASUKAWA M., SUETAKE T., 1973. Studies on the retention of gametes of salmonids fishes. I. - Oh the fertility of chum salmon eggs after storage. Scient. Rep. Hokkaido Salmon Hatchery, 27, 31-37.

TRUSCOTT B., IDLER D. R., HOYLE R. J., FREEMAN H. C., 1968. Subzero preservation of Atlantic salmon sperm. J. Fish. Res. Bd. Can., 25, 363-372.

WITHLER F. C., MORLEY R. B., 1968. Effects of chilled storage on viability of stored ova and sperm of sokeye and pink salmon. J. Fish. Res. Bd. Can., 25, 2695-2699. 